\title{
Creencias adolescentes sobre la violencia de género. Sexismo en las relaciones entre adolescentes
}

\author{
Zoraida de la Osa Escudero ${ }^{1}$, Soledad Andrés Gómez ${ }^{2}$ e Isabel \\ Pascual Gómez ${ }^{2}$ \\ ${ }^{1}$ Colegio Gredos San Diego Alcalá de Henares, ${ }^{2}$ Universidad de Alcalá de Henares
}

(España)

El presente trabajo indaga en las relaciones de pareja en la adolescencia, analizando las creencias de los y las adolescentes atendiendo al factor violencia de género desde una perspectiva cognitivo-evolutiva. La muestra está compuesta por 297 estudiantes con edades comprendidas entre los 13 y 18 años $\left(2^{\circ}\right.$ ESO, $4^{\circ}$ ESO y $2^{\circ}$ Bachillerato) de tres centros privados-concertados de Madrid. Analizamos sus representaciones de las relaciones de pareja desde el punto de vista de ambos géneros y su grado de acuerdo con la justificación de violencia. El estudio responde a un diseño transversal (cuasi-experimental) con encuesta. El instrumento utilizado es una adaptación del Cuestionario de Actitudes hacia la Diversidad y la Violencia (CADV). Los resultados muestran una correspondencia parcial en la relación entre el desarrollo evolutivo-moral y el grado de justificación de diferentes situaciones en las que se presenta la violencia. De acuerdo con nuestros datos, el acuerdo con creencias sexistas y justificación de la violencia desciende según se avanza en la escolarización. Al mismo tiempo, obtenemos que es en el tramo de edad de quince a dieciséis años ( $\left.4^{\circ} \mathrm{ESO}\right)$ donde justifican más los estereotipos de género. Todo ello nos lleva a señalar la adolescencia media como curso diana en el que realizar una intervención psicoeducativa considerando los valores relacionados con la igualdad y el respeto entre los géneros.

Palabras clave: Adolescencia, violencia de género, diferencias de género, intervención psicoeducativa.

Beliefs about gender violence. Sexism in adolescent relationships. This paper explores the relationships in adolescence, examining beliefs about gender violence from a cognitivedevelopmental perspective. The sample consists of 297 students from 3 different statesubsidized schools, between the ages of 13-18 (2nd and 4th years of Secondary Education and 2nd year of High School). The students' representations of relationships are analyzed from the point of view of both genders and the degree to which they consider violence to be justified. The study is based upon a transversal design (quasi-experimental) with survey. The instrument used is an adaptation of the Cuestionario de Actitudes hacia la Diversidad y la Violencia (Questionnaire of Attitudes towards Diversity and Violence). The results demonstrate a partial relationship between moral-evolutionary development and the degree of justification applied to different situations in which there is violence. The data shows that the acceptance of sexist beliefs and the justification of violence decrease as students get older. However, from 15-16 years old (4th ESO) is the age at which students most justify gender stereotypes. The study indicates middle- adolescence as the ideal time to carry out psycho-educational interventions, taking into account the values related to equality and respect between genders.

Key words: Adolescence, gender violence, gender differences, psycho-educational intervention.

Correspondencia: Zoraida de la Osa Escudero. Departamento de Orientación, Colegio Gredos San Diego Alcalá de Henares. Avda. Víctimas del Terrorismo, 7 D, ático B. C.P. 28806. Alcalá de Henares, Madrid (España). E-mail: zoraidadelaosa@gmail.com 
Numerosos organismos e instituciones internacionales (Organización de Naciones Unidas y sus diversos organismos) y nacionales (Instituto Nacional de Estadística e Instituto de la Mujer (2010, 2009, 2008, 2007, 2006) y el Observatorio Estatal de Violencia contra la mujer $(2007,2010)$ informan de datos que justifican la violencia contra la mujer como un problema que atañe a la sociedad contemporánea. Así lo constata el informe publicado por el Instituto de la Mujer (2006), en el que se analizan los resultados de Macroencuestas sobre la violencia contra las mujeres (1999, 2002, 2006) con mujeres de 18 en adelante. Comparando los datos de los diferentes años observamos un descenso tanto en el maltrato técnico [Según la definición del informe, el maltrato técnico se refiere a las mujeres que, aunque no se consideren a sí mismas como maltratadas, responden que son víctimas de determinados comportamientos, considerados como indicativos de cierto grado de violencia, por las personas expertas] (12.4\% en $1999,11.1 \%$ en $2002,9.6 \%$ en 2006) como en el maltrato declarado [Maltrato declarado: mujeres que confiesan haber sido maltratadas durante el último año] (4.2\% en 1999, 4\% en 2002 y 3.6\% en 2006). Por su parte, la Comisión para la Investigación de Malos Tratos (2005) informa de la incidencia del problema con mujeres de edades comprendidas entre los 16 y 30 años. Así, existe un 39.8\% de denuncias de mujeres víctimas de violencia de género, siendo el $32.7 \%$ feminicidios en el ámbito de la pareja.

En nuestro estudio asumimos la definición de violencia propuesta por Del Barrio (2003), que parte del concepto global de conducta antisocial. La categoría más amplia sería conductas antisociales, en la que se incluye la agresión como categoría más específica, pero también otras conductas dirigidas a ocasionar un daño, no a otros como individuos, sino a las instituciones sociales. La agresión es la conducta que se propone infligir un daño físico o psicológico a otro individuo. Las definiciones de violencia suelen aludir a fenómenos de destrucción, fuerza y coerción que ocurren en las relaciones, la sociedad o incluso en la naturaleza. Las premisas que tenemos en consideración para determinar la violencia son: 1) que esa fuerza o vehemencia sea física y 2) "actos, actuaciones, destructores de una realidad, propia o ajena. Entendiendo que en la violencia entra el hacer, pero también el decir, el mirar o el desear; que la realidad que se destruye no es física y material, sino que también se destruyen los afectos, las conciencias, la convivencia” (Del Barrio et al., 2003, p. 15).

Diferentes ramas de la Psicología (Social, Educativa y Evolutiva), las Ciencias de la Salud y la Sociología son las disciplinas que más han destacado en el estudio de la violencia de género. Kanin (1957) y, posteriormente, Makepeace (1981, 1986) mostraron su interés en este ámbito, siendo pioneros en el estudio de la naturaleza y prevalencia de la violencia en el noviazgo. En España, el equipo de Díaz-Aguado ha realizado diferentes estudios con el fin de desarrollar programas de prevención de la violencia en la adolescencia, señalando entre sus conclusiones la existencia de una 
asociación significativa entre el nivel de razonamiento menos desarrollado y el elevado acuerdo con las creencias que niegan el valor de la igualdad y la tolerancia (sexismoviolencia de género). Otros resultados de este equipo muestran la existencia de importantes diferencias en el razonamiento moral sobre la violencia hacia las mujeres en función del género (Díaz-Aguado, 2002, 2003, 2004): la mayoría de las y los adolescentes parecen rechazar las creencias y estereotipos sexistas, así como las que justifican la violencia contra la mujer, al tiempo que reconocen que este problema afecta al conjunto de la sociedad. Además, las creencias sexistas y de justificación de la violencia son rechazadas en mayor grado por las chicas (entre el $70 \%$ y el $98 \%$ ) que por los chicos (43\% al 86\%). Sin embargo, este reconocimiento es compatible con la justificación de la agresión en las relaciones interpersonales ante ofensas recibidas, o con la idea de que alguien hubiera atentado contra quien -la chica, novia, mujer- que considerasen de su propiedad.

Recientemente, el macro-estudio Igualdad y Prevención de la Violencia de Género en la Adolescencia (Díaz-Aguado, 2011) señala que el $74.1 \%$ de las chicas se encuentran protegidas frente a la violencia de género, y el $64.7 \%$ de los chicos frente al riesgo de ejercerla. No obstante, se detectan más víctimas (4.9\%) que agresores $(4.2 \%)$, señalándose que las situaciones de maltrato más frecuentes están relacionadas con el control emocional y conductas derivadas. De interés también resulta el que las preguntas que indagan por los valores buscados en las relaciones de pareja obtengan resultados similares a los de diez años atrás, mostrando la persistencia y pervivencia de estereotipos, en especial entre los chicos.

\section{Objetivos}

El objetivo general es conocer las relaciones de pareja en la adolescencia analizando las expectativas y creencias de los y las adolescentes, atendiendo al factor violencia de género. De él se derivan los siguientes objetivos específicos:

1) Conocer las representaciones de las relaciones de pareja que tienen chicos y chicas desde el punto de vista de ambos géneros, en edades comprendidas entre los 13 y 16 años.

2) Establecer el grado de acuerdo con la justificación de violencia doméstica.

3) Establecer el grado de rechazo de la violencia.

\section{Hipótesis}

La hipótesis general de nuestro estudio es la existencia de relación entre el desarrollo evolutivo moral y las creencias sexistas y de justificación de la violencia doméstica, la justificación de la violencia entre iguales ante ofensas recibidas o la idea de que alguien ha atentado contra quien consideran de su propiedad, y el grado de 
acuerdo con creencias tolerantes y de rechazo de la violencia. A partir de ella, establecemos hipótesis de carácter específico:

Hipótesis 1.1. La justificación de la violencia entre iguales como reacción y valentía correlaciona negativamente con la edad/curso.

Hipótesis 1.2. El acuerdo con creencias tolerantes y de rechazo de la violencia, correlaciona de manera positiva con la edad/curso.

Hipótesis 1.3. Las creencias sexistas y de justificación de la violencia doméstica correlacionan negativamente con el entorno sociocultural y socioeconómico de los centros, y también con el curso.

Hipótesis 1.4. Las chicas muestran mayor grado de acuerdo con creencias tolerantes y de rechazo de la violencia.

Hipótesis 1.5. Los chicos justifican en mayor medida la violencia entre iguales como reacción y valentía, manteniendo un mayor grado de acuerdo con creencias sexistas y de justificación de la violencia que las chicas.

\section{MÉTODO}

\section{Diseño}

Siguiendo la perspectiva teórica expuesta, el diseño de evaluación es transversal (cuasi-experimental) con encuesta.

\section{Participantes}

La muestra está compuesta por 297 chicos y chicas con edades comprendidas entre los 13 y 18 años ( $2^{\circ} \mathrm{ESO}, 4^{\circ}$ ESO y $2^{\circ}$ Bachillerato) de tres centros privados concertados laicos de la provincia de Madrid (Tabla 1).

Tabla 1. Participantes por curso y centro

\begin{tabular}{ccccc}
\hline & Centro 1 & Centro 2 & Centro 3 & Total CURSO \\
\hline $2^{\circ}$ ESO & 48 & 22 & 17 & 87 \\
\hline CHICOS & 20 & 8 & 8 & 36 \\
\hline CHICAS & 28 & 14 & 9 & 51 \\
\hline $4^{\circ}$ ESO & 29 & 45 & 55 & 129 \\
\hline CHICOS & 20 & 27 & 23 & 70 \\
\hline CHICAS & 9 & 18 & 32 & 59 \\
\hline $2^{\circ}$ BACH & 15 & 31 & 35 & 81 \\
\hline CHICOS & 5 & 19 & 12 & 36 \\
\hline CHICAS & 10 & 12 & 23 & 45 \\
\hline Total CENTRO & 92 & 98 & 107 & 297 \\
\hline
\end{tabular}

\section{Instrumentos}

El instrumento utilizado es una adaptación del Cuestionario de Actitudes hacia la Diversidad y la Violencia $(C A D V)$, utilizado para conocer los componentes afectivos y cognitivo de las actitudes relacionadas con justificación de la violencia y las 
creencias sexistas. Se trata de una escala de tipo Likert de elección múltiple (de 1 a 7) con 40 ítems. Se estructura en tres factores: Justificación de la violencia entre iguales como reacción y valentía (Factor I) de 16 ítems; Creencias sexistas y justificación de la violencia doméstica (Factor II) compuesto por 17 ítems y Acuerdo con creencias tolerantes y de rechazo de la violencia (Factor III) de 7 ítems.

La versión adaptada del CADV confirma la estructura factorial de la versión original. Así, los coeficientes de fiabilidad (Alpha de Cronbach) encontrados fueron: $\alpha=0.832$ para la prueba total; $\alpha=0.807$ en el factor Justificación de la violencia entre iguales como reacción y valentía; $\alpha=0.806$ en el factor Creencias sexistas y justificación de la violencia doméstica y $\alpha=0.639$ en el factor Acuerdo con creencias tolerantes y de rechazo de la violencia.

\section{Procedimiento}

Antes de iniciar el proceso de recogida de datos se presentó en cada centro implicado una presentación del proyecto y se realizó una entrevista con cada uno de los directores de los tres centros educativos participantes en el estudio. El objetivo era doble, por una parte ofrecerles la información relativa al desarrollo del proyecto y por otra, lograr su implicación y colaboración. A su vez ellos pusieron esta información en conocimiento de los respectivos Equipos Directivos y Jefaturas de Estudios. Una vez aprobada la realización del proyecto en cada centro, se acordó realizar la recogida de datos en junio de 2010.

\section{El tratamiento de datos}

El análisis de los resultados de los cuestionarios se ha realizado utilizando el programa estadístico SPSS versión 17.0 para Windows. El tratamiento estadístico realizado fue un análisis de varianza (ANOVA) con el objetivo de conocer la presencia de diferencias significativas en función del género y del curso.

\section{RESULTADOS}

Los principales resultados encontrados nos permiten responder a las siguientes preguntas: ¿Los adolescentes justifican la violencia entre iguales como reacción y valentía? ¿Participan de creencias sexistas y justificación de la violencia doméstica? O por el contrario, ¿manifiestan creencias tolerantes y de rechazo de la violencia?

Los resultados generales (Gráfica 1) muestran que, aunque en mayor medida los y las adolescentes participan de creencias tolerantes y de rechazo de la violencia (4.92), continúa existiendo cierto grado de acuerdo (2.44) con afirmaciones que justifican la violencia como conducta de reacción y valentía, así como con creencias sexistas y justificación de la violencia doméstica (2.04). 
Gráfica 1. Medidas generales por factores

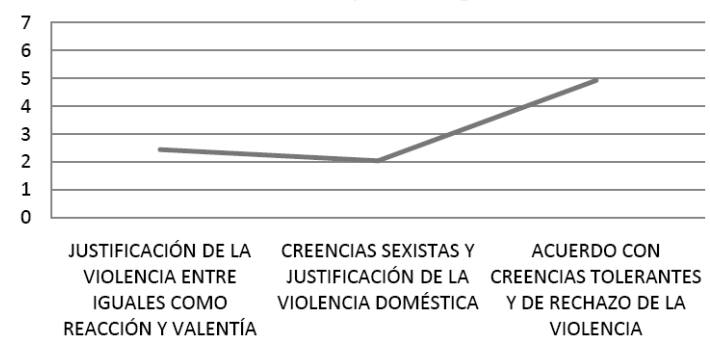

Respecto a la evolución por cursos si bien la justificación de la violencia debería ir disminuyendo cuando el alumno se encuentra en cursos superiores como ocurre en el factor I, observamos (Gráfica 2) que es en $4^{\circ}$ de ESO donde justifican más la violencia entre iguales como conducta de reacción y valentía (Media=2.46; sig=0.000).

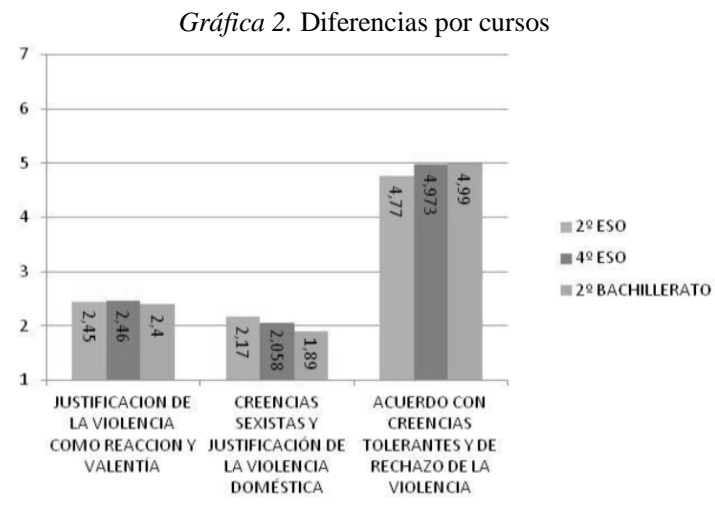

Gráfica 3. Diferencias por género

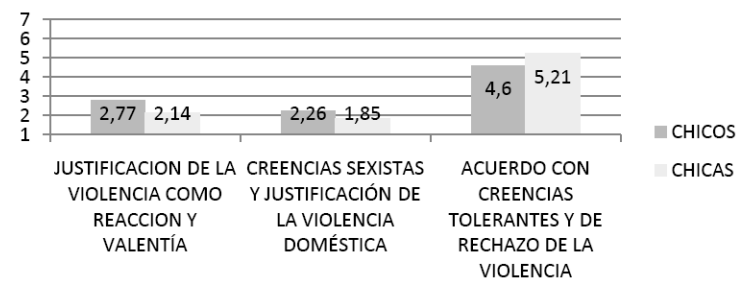

¿Existen diferencias de género en los factores estudiados? Atendiendo a la variable género los resultados obtenidos en el estudio (Gráfica 3) muestran diferencias significativas en los tres factores estudiados (Factor 1 sig=0.01; Factor 2 sig=0.007; Factor 3 sig=0.00). Son los chicos quienes en mayor grado justifican la violencia ante ofensas recibidas o la idea de que alguien ha atentado contra quien consideran de su 
propiedad (Media=2.77) y presentan mayor acuerdo con creencias sexistas y justificación de la violencia doméstica (Media=2.26). A pesar de que el acuerdo con creencias tolerantes y de rechazo de la violencia es alto, es mayor el de las chicas (Media=5.21) que el de los chicos (Media=4.60).

¿Cómo se muestran las diferencias destacadas anteriormente en los cursos a los que pertenece la muestra? Encontramos que son los chicos quienes en mayor grado justifican la violencia como reacción y valentía, y el grado con dicha justificación es mayor cuanto mayor es el curso al que pertenecen (Gráfica 4) llegando a un grado de acuerdo de 2.82 en $2^{\circ}$ Bachillerato. Por otro lado, el acuerdo con creencias tolerantes y de rechazo de la violencia es mayor en las chicas que en los chicos. Además, atendiendo a este factor, encontramos que el grado de acuerdo aumenta en las chicas conforme ascendemos de curso; por el contrario, mientras que el grado de acuerdo es mayor en los chicos de $4^{\circ}$ ESO (4.73) que en los de $2^{\circ}$ ESO (4.62), no es así en $2^{\circ}$ Bachillerato; pues son estos últimos quiénes participan en menor grado de acuerdo (4.34) con creencias tolerantes y de rechazo de la violencia.

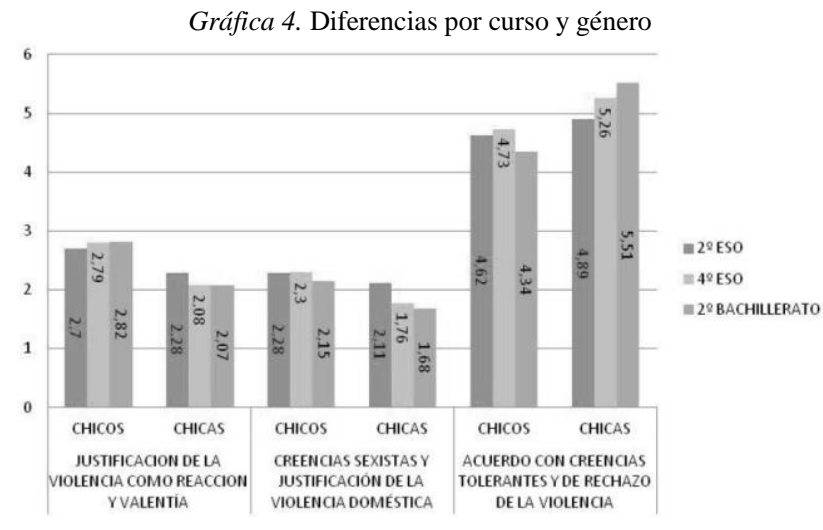

\section{CONCLUSIONES}

La mayoría de adolescentes parecen rechazar las creencias y estereotipos sexistas, así como las que justifican la violencia contra la mujer, reconociendo que este problema afecta al conjunto de la sociedad, de acuerdo con los datos de estudios precedentes (Díaz-Aguado, 2010, 2004, 2002, 2001; González, 2008; Toldos, 2002; González, 2001; Instituto de la Mujer, 2006, 2002, 1999). La hipótesis de partida de este trabajo sugiere que, siguiendo las teorías cognitivo-evolutivas, por un lado existe relación entre el desarrollo evolutivo-moral y el grado de justificación de diferentes situaciones en las que se presenta la violencia; y, por otro, el acuerdo con creencias 
tolerantes. No obstante, nosotras hemos encontrado correspondencia tan sólo parcial con los resultados de nuestro estudio.

En primer lugar, de nuestros datos se desprende que el curso al que pertenece el alumnado está relacionado con el grado de acuerdo que presentan con creencias tolerantes y de rechazo de la violencia; al igual que en otros estudios (Díaz-Aguado, 2001, 2003, 2004), en donde dicho acuerdo aumenta con la edad. Al mismo tiempo, a más edad, disminuye el grado de acuerdo que los alumnos y alumnas tienen con creencias sexistas y de justificación de la violencia doméstica, como muestran cuando afirman que el problema de la violencia contra las mujeres por parte de sus maridos o compañeros afecta al conjunto de la sociedad. Sin embargo, es en el tramo de edad de quince a dieciséis años (correspondiente al alumnado escolarizado en su mayoría en $4^{\circ}$ ESO) en donde más justifican la violencia entre iguales ante ofensas recibidas o la idea de que alguien ha atentado contra quien consideran de su propiedad, al mostrar un alto grado de acuerdo con afirmaciones como "es correcto pegar a alguien que te ha ofendido, se debe infundir miedo a algunas personas para que te respeten, el hombre que parece agresivo es más atractivo y la mujer que parece débil es más atractiva”.

Así, nuestros resultados coinciden con otros precedentes en el campo, en donde se ha encontrado una relación entre esta dimensión y la asociación que los adolescentes realizan con una identidad basada en los estereotipos de género (Toldos, 2002; Díaz-Aguado, 2001). De tal forma que son los alumnos y alumnas de $4^{\circ}$ ESO quienes justifican, en mayor medida, los estereotipos de género en comparación con los cursos de $2^{\circ}$ ESO, alumnado más joven (12-13 años), y, en sentido contrario, de $2^{\circ}$ Bachillerato, alumnado dos años mayor que el grupo que nos ocupa (17-18 años). El valor de este dato nos induce a señalar este período de la adolescencia media como curso diana en el que adquiere especial importancia el trabajo psicoeducativo para el desarrollo de los valores relacionados con la igualdad y el respeto entre los géneros.

Atendiendo al análisis curso y género, nuestros datos no nos han mostrado diferencias entre chicos y chicas en ninguna de las dimensiones estudiadas, excepto en la que hace referencia a las creencias tolerantes. De acuerdo con ellos, los varones justifican en mayor grado la violencia al referirse a la idea de que alguien ha atentado contra quien consideran de su propiedad, y la violencia doméstica, explicitando un mayor acuerdo con creencias sexistas que las chicas del estudio. Vemos cómo hoy en día continúa existiendo una diferenciación significativa de expectativas y creencias en nuestros adolescentes relacionadas con el sexismo y la justificación de la violencia. Sobre todo, los chicos continúan participando de los estereotipos de género y justificando la división de roles según el sexo, identificando valores como el ejercicio de la responsabilidad, la concepción del trabajo como expresión de la autonomía económica y como proyecto personal a lo largo de la vida, el carácter decidido, fuerte y valiente (o sumiso y no competitivo), la capacidad para pedir y ofrecer ayuda, la disponibilidad para 
el cuidado de niños y mayores, etc., con uno u otro género de acuerdo con las ideas sociales más tradicionales.

Por último, lo que parece mostrar nuestro estudio - de nuevo de acuerdo con los precedentes del campo ya mencionados-, es que los y las adolescentes necesitan continuar avanzando en la superación del sexismo y la violencia de género. Pero, ¿cómo ayudarles en este progreso? Uno de los contextos más cercanos a nuestros chicos y chicas y con un papel esencial en el desarrollo social y moral de la personalidad adolescente, que permitiría la superación de estos problemas, es la escuela. No por poco repetido deja de seguir siendo necesario insistir en ello, como muestran, de nuevo, los resultados de nuestro trabajo. Ahora bien, ¿está formada -en las concepciones del profesorado y en la práctica curricular- y organizada -en su dimensión temporal y espacial- la escuela para guiar y apoyar a los y las adolescentes, promoviendo así el cambio generacional esperado por la sociedad? No parece que sea sencillo llevar a la práctica el trabajo de desarrollo social que la tarea requiere, como señala Díaz-Aguado (2010). De acuerdo con ella, resulta imprescindible el impulso de los programas de prevención de la violencia desde el ámbito socioeducativo. Su objetivo, como no puede ser de otra manera, estará al servicio de la promoción de un modelo de relaciones entre hombres y mujeres basado en la igualdad y respeto mutuo, como modelo general de convivencia en consonancia con el respeto a los derechos humanos que exige la sociedad actual. En el mismo sentido, es también sabido que la familia es el primer contexto de socialización, siendo clave su papel en la formación en valores. Así que, a la pregunta sobre el papel de la familia con respecto a la superación del sexismo y la violencia de género, la respuesta está en la necesidad del desarrollo de tareas de educación conjunta en ambos contextos. Por un lado, porque es imprescindible formar a los y las adolescentes en modelos no sexistas de las relaciones interpersonales y, por otro, porque la colaboración conjunta en la formación en valores como la igualdad, tolerancia y respeto ve multiplicada su influencia educativa. Además, en el medio social más amplio, se debe formar en el análisis crítico de los modelos de relaciones interpersonales que ofrecen los medios de comunicación, en concreto, programas televisivos en los que la tolerancia y el respeto resultan inexistentes.

Por último, se presentan algunas consideraciones acerca de la necesidad de profundizar el estudio en este ámbito a partir de mejoras en la metodología de investigación. El uso de muestras de mayor tamaño, por una parte, y, por otra, la inclusión de instrumentos cualitativos como grupos de discusión y entrevistas en profundidad, permitirían conocer mejor las características del problema, sobre todo, en las edades que parecen presentarse como grupos de riesgo. Parece pertinente, asimismo, estudiar la influencia de las características del entorno familiar (tipo de relaciones que se establecen, valores que se transmiten) y escolar (ídem). En definitiva, profundizar en el estudio de las causas para poder avanzar en la superación del sexismo y con ello, 
establecer los objetivos de una intervención orientada a la modificación de creencias sexistas en la adolescencia.

\section{REFERENCIAS}

Comisión para la Investigación de Malos Tratos a Mujeres (2005). Informe La Violencia de Género en Mujeres Jóvenes. http://www.malostratos.org.

Del Barrio, C. y Martín, E. (2003). Convivencia y conflicto en los centros escolares: introducción. Infancia y Aprendizaje, 26(1), 5-8.

Del Barrio, C., Martín, E., Almeida, A. y Barrios, A. (2003). Del maltrato y otros conceptos relacionados con la agresión entre escolares, y su estudio psicológico. Infancia y Aprendizaje, 26(1), 9-24.

Díaz-Aguado, M.J. y Martínez, R. (2001). La construcción de la igualdad y la prevención de la violencia contra la mujer desde la educación secundaria. Madrid: Instituto de la Mujer.

Díaz-Aguado, M.J. (2002). Prevenir la violencia contra las mujeres construyendo la igualdad. Madrid: Instituto de la Mujer.

Díaz-Aguado, M.J. (2004). Prevención de la violencia y lucha contra la exclusión desde la adolescencia. Madrid: Instituto de la Juventud.

Díaz-Aguado, M.J. y Carvajal, M.I. (2010). Estudio estatal sobre la convivencia escolar en la Educación Secundaria Obligatoria. Madrid: Ministerio de Educación.

González, M.P. (2008) Violencia en las relaciones de noviazgo entre jóvenes y adolescentes de la Comunidad de Madrid. Tesis doctoral no publicada. Universidad Complutense de Madrid.

González, R. y Santana, J.D. (2001). La violencia en parejas jóvenes. Psicothema, 13(1), 127-131.

Instituto de la Mujer (2006). III Macroencuesta sobre la violencia contra las mujeres. Madrid: Instituto de la Mujer. Ministerio de Trabajo y Asuntos Sociales.

Instituto de la Mujer (2008). Las mujeres en cifras 1983-2008. Madrid: Instituto de la Mujer. Ministerio de Igualdad.

Instituto Nacional de Estadística e Instituto de la Mujer (2010). Mujeres y hombres en España 2010. Madrid: INE.

Instituto Nacional de Estadística e Instituto de la Mujer (2009). Mujeres y hombres en España 2009. Madrid: INE.

Instituto Nacional de Estadística e Instituto de la Mujer (2008). Mujeres y hombres en España 2008. Madrid: INE.

Instituto Nacional de Estadística e Instituto de la Mujer (2007). Mujeres y hombres en España 2007. Madrid: INE.

Instituto Nacional de Estadística e Instituto de la Mujer (2006). Mujeres y hombres en España 2006. Madrid: INE.

Kanin, E.J. (1957). Male Aggression in Dating-Courtship Relations. The American Journal of Sociology, 63(2), 197-204.

Makepeace, J.M. (1981). Courtship violence among college students. Family Relations, 30, $97-$ 102.

Makepeace, J.M (1986). Gender Differences in Courtship Violence Victimization. Family Relations, 35(3), 383-388.

Ministerio de Trabajo y Asuntos Sociales (2007). II Informe Anual de Observatorio Estatal de Violencia sobres la Mujer. Madrid: Ministerio de Trabajo y Asuntos Sociales. http://www.observatorioviolencia.org/upload_images/File/DOC1184747918_Informe\%2 0Anual\%2028\%20junio\%202007.pdf 
Ministerio de Sanidad Política e Igualdad (2010). III Informe Anual del Observatorio Estatal de Violencia sobre la Mujer. Madrid: Ministerio de Sanidad, Política Social e Igualdad. http://www.migualdad.es/ss/Satellite?cid=1193047406913\&pagename=MinisterioIguald ad\%2FPage\%2FMIGU_contenidoFinal

Organización de Naciones Unidas (1975). I Conferencia sobre la mujer. México: Editor.

Organización de Naciones Unidas (1975). II Conferencia sobre la mujer. Copenhague: Editor.

Organización de Naciones Unidas (1985). III Conferencia Internacional sobre la mujer. Nairobi: Editor.

Organización de Naciones Unidas (1985). IV Conferencia Internacional sobre la mujer. Beijing: Editor.

Organización de Naciones Unidas (1993). II Congreso por los Derechos Humanos. Viena: Editor.

Organización de Naciones Unidas (1994). Declaración sobre la eliminación de la violencia contra la mujer. Resolución de la Asamblea General 48/104 del 20 de diciembre de 1993. Ginebra: Editor.

Toldos, M. (2002) Adolescencia, Violencia y Género. Tesis doctoral no publicada. Universidad Complutense de Madrid.

Recibido: 13 de febrero de 2013

Recepción Modificaciones: 8 de marzo de 2013

Aceptado: 22 de agosto de 2013 